\title{
Hemodynamically unstable/ shocked patient in OR challenge for teamwork efficacy when time is critical
}

Volume 7 Issue 4 - 2017

\section{Opinion}

In the present article we present a case report with comments/ suggestions about team dynamics. The emergency case report is only used to highlight the importance of teamwork dynamics and many clinical details are not mentioned. Treating a hemodynamically unstable/shocked patient can be a challenging procedure and requires perfect teamwork to have the best possible outcome. Reviewing the present case report we should always keep in mind that the organization is a private hospital with all the differences in resources compared to a large public hospital.

A 33-year old female patient presented to ER department with signs of circulatory shock (low blood pressure, tachycardia, drowsiness and clumsy sweaty skin). Her vital signs were $\mathrm{BP}=75 / 35 \mathrm{mmHg}$, HR ranging from 120-135 bpm, temperature was 35.9 Celsius, pale skin and drowsiness. Initial resuscitation in ER included administration of 2 liters IV ringers lactate and oxygen through face mask at 5-6 lt/ $\mathrm{min}$. There was temporary improvement of vital signs. Bloods were sent for complete blood count, coagulation, biochemistry.

Patient had a painful distended abdomen and $\mathrm{Ob} / \mathrm{Gynae}$ doctors were called. Ultrasound of the abdomen revealed presence of fluid in the abdomen and in combination with medical history and clinical exam lead to the possible diagnosis of a ruptured ectopic pregnancy. Results of the blood workout can be seen in Table 1 .

Table I Basic results of patients' blood investigation in emergency department

\begin{tabular}{ll}
\hline Investigation & Value \\
\hline $\mathrm{Hb}$ & $8.5 \mathrm{~g} / \mathrm{dl}$ \\
$\mathrm{Ht}$ & $26 \%$ \\
$\mathrm{PLT}$ & $210 \times 10^{3}$ \\
$\mathrm{INR}$ & 1.35 \\
$\mathrm{aPTT}$ & 44
\end{tabular}

Patient was urgently transferred to $\mathrm{OR}$ for an emergency laparotomy. Patient had one IV cannula $18 \mathrm{G}$ in left antecubital fossa. Two more $16 \mathrm{G}$ cannula were inserted in both hands before anesthesia induction. An infusion of phenylephrine $(50 \mathrm{mcg} / \mathrm{ml})$ was started at a rate of $5 \mathrm{ml} / \mathrm{h}$ and titrated to effect (target to keep mean arterial pressure around $60 \mathrm{mmHg}$ ).

The induction of anesthesia was performed in semi-sitting position to minimize risk of aspiration with $5 \mathrm{mg}$ midazolam IV and $20 \mathrm{mg}$ etomidate IV. When patient lost consciousness, $12 \mathrm{mg}$ cisatracurium was administered IV and after 2 minutes' patient intubated uneventful. Blood bank informed that packed RBC would be ready in 20 min. Fluid resuscitation continued, phenylephrine infusion titrated to keep a mean arterial pressure between $50-60 \mathrm{mmHg}$ and $1 \mathrm{gr}$ tranexamic given IV. Despite all resuscitation effort, blood pressure was always borderline. Arterial blood gases and new $\mathrm{CBC}$ and coagulation sent. The intraoperative results regarding $\mathrm{CBC}$ and coagulation can be seen in Table 2.

\author{
Dimosthenis Petsas, I Yasir Othman AT, I \\ Marton Hannus, I Eljona Cumashi2 \\ 'Anaesthetics Department, HMG Hospital, UAE \\ 2OB/GYNAE Department, HMG Hospital, UAE
}

Correspondence: Dimosthenis Petsas, Anaesthetics Department, HMG Hospital Dubai, Dubai Healthcare City, Building 57, UAE, PO BOX 505005, Tel 00306972008383 , Email dpets_anesthesia@hotmail.com

Received: March 10, 2017 | Published: March 15, 2017

Table 2 Intraoperative hemoglobin and coagulation

\begin{tabular}{ll}
\hline Investigation & Value \\
\hline $\mathrm{Hb}$ & $4.1 \mathrm{~g} / \mathrm{dl}$ \\
$\mathrm{PLT}$ & $140 \times 10^{3}$ \\
$\mathrm{INR}$ & 1.6
\end{tabular}

From the blood results, it is obvious that patient had massive blood loss (intraoperative blood loss was approx. 3 lt) that possibly was tamponating the vessels by increased intraabdominal pressure before surgical intervention. We can also see the initiation of coagulation abnormalities (although the patient had not reached the diagnostic criteria of disseminated intravascular coagulation). Surgical intervention was fast and efficient controlling the bleeding. Transfusion started with two units of packed red blood cells plus 1 unit of fresh frozen plasma (FFP). After 2 units of packed RBC and $1 \mathrm{FFP}$, hemoglobin rose to $6.5 \mathrm{~g} / \mathrm{dl}$. Patient transferred intubated to ICU where transfusion continued. Patient extubated next day and her postoperative recovery was uneventful. This short scenario is used as a paradigm to highlight good team dynamics. We can see the team dynamics in several phases:

i. Before transferring patient to OR: Patient evaluated fast in ED by anesthetist and anesthetist informs OR team/Anesthesia technician about induction technique, medication IV fluids and monitoring, so when patient arrives to OR, Anesthetic technician will have all this ready and he can help rest of the team. Also, anesthetist and surgeon informed OR nurses about procedure details so they can be ready before patient arriving to OR.

ii. Coming to OR: While resuscitation still ongoing, all team participate positioning patient and anesthesia team with ED nursing team connecting monitors to avoid any gap in monitoring the unstable patient.

iii. When patient inside OR: connecting to monitors while surgical team getting ready. 
iv. Recovery nurse (free person) allocated to be the connection between anesthetist, laboratory and blood bank (to be the free person able to communicate continuously/avoid interruptions in ongoing resuscitation by anesthesia team)

v. When surgical team ready, induction of anesthesia and start operation.

vi. Continuous verbal communication between surgical team and anesthetic team.

\section{vii. Informing ICU.}

The dilemma of more complicated invasive monitoring must always be weighted against critical time. We strongly believe that the key points of good outcome in this case was the non-interruption of resuscitation by allocating recovery nurse as communication link with blood bank and lab that otherwise would be part of the anesthetist duty.

Also, the communication and preparation of OR team before patient arriving. Preparation of nurses and anesthetic technician while patient inside OR would result in a critical waste of time and interruption of resuscitation.
A well communicated and verbalized plan to all OR staff before an unstable enters OR is of paramount importance to maximize possibility of good outcome. With the above-mentioned case and comments anyone can understand the importance of clinical leadership and communication in our everyday practice. While this case and comments probably are not new knowledge it is always useful to remind us the importance of communication and team work in an interesting format of a case report.

\section{Conflicts of interest}

There is no conflict of interest.

\section{Acknowledgements}

None.

\section{Funding}

None. 